\title{
Data processing and optimization system to study prospective interstate power interconnections
}

\author{
Sergei Podkovalnikov ${ }^{1, *}$, Ivan Trofimov ${ }^{1}$, and Leonid Trofimov ${ }^{1}$ \\ ${ }^{1}$ Melentiev Energy Systems Institute Siberian branch of the Russian academy of sciences, Department \\ of Electric Power Systems. 664033, Irkutsk, Lermontov St., 130. Russia
}

\begin{abstract}
The paper presents Data processing and optimization system for studying and making rational decisions on the formation of interstate electric power interconnections, with aim to increasing effectiveness of their functioning and expansion. The technologies for building and integrating a Data processing and optimization system including an object-oriented database and a predictive mathematical model for optimizing the expansion of electric power systems ORIRES, are described. The technology of collection and pre-processing of non-structured data collected from various sources and its loading to the object-oriented database, as well as processing and presentation of information in the GIS system are described. One of the approaches of graphical visualization of the results of optimization model is considered on the example of calculating the option for expansion of the South Korean electric power grid.
\end{abstract}

\section{Introduction}

The relevance of research in the field of expansion of interstate power interconnections is determined by the expansion of globalization processes in the world. To meet electricity demands, there is a need to integrate electricity systems both within the country and among countries. Such interconnections are already functioning in Europe, North and South America and other regions of the world [1]. However, in order to justify the creation of such interconnections, qualitative preliminary calculations are required to predict the effectiveness, ensure the reliability of the interconnected power systems, and much more.

Conducting scientific research in this area requires a lot of preliminary work on the collection and analysis of a huge amount of information, complex technical and economic calculations, significant intellectual and information resources. Without the presence of advanced information technologies, specialized in this subject area, research takes a huge amount of time and money. Multidimensional results of calculations are also difficult to imagine without a specialized graphical interface for data representation and processing.

The Data Processing and Optimization System (DPOS), is being developed by the authors, integrates in itself the technologies for collecting and processing non-structured data from open sources; the technology of information restructuring in the object view; the

* Corresponding author: spodkovalnikov@isem.irk.ru 
technology of graphical and cartographic data representation in the GIS system; and the mathematical model for optimizing the expansion and operating modes of the power systems/interconnections - ORIRES. As a result of the DPOS usage, the efficiency and quality of scientific research is increased in the field of expansion of interstate electric power systems being conducted at the Melentiev Energy Systems Institute of the Siberian Branch of the Russian Academy of Sciences (ESI SB RAS).

\section{The data processing and optimization system architecture}

In ESI SB RAS conducted is research into the possibilities of combining national power systems of various countries (mainly in the Northeast Asia region) into interstate power interconnections. The authors are developing the DPOS for complex information and analytical support of scientific research in the field of expansion of interstate energy interconnections. At calculations the model for optimizing expansion and modes of electric power systems ORIRES, integrated in DPOS is used.

The DPOS is based on an object-oriented database (OODB) [2] to store and processing of information. All information in the OODB is structured as objects. Types of objects in the OODB are independent and different - power plant, city, region, country, power interconnection, power line and others. Each object is a record containing a unique object identifier, a set of parameters describing it, and the values of each parameter in text and numeric form, which can be stored by year.

A user interface for inputting / outputting information from OODB has been developed, using graphical and cartographic representation of energy/power data. For the presentation of materials, combined fragments of GIS maps with the main components of the electric power infrastructure are used. The information can be presented in retrospect from the $90 \mathrm{~s}$ to the present, and also taking into account the expected medium and long-term outlook.

An interface is also being developed to work with the optimization model ORIRES integrated into DPOS to perform complex optimization studies of various scenarios for the formation of interstate power interconnections, including studies of the prospects for the creation of the Asian Energy Supergrid [3-4].

The main structural-functional blocks of DPOS, Fig. 1:

- Procedures (technologies) for collecting information resources,

- Object-oriented database,

- Interface for working with the mathematical model ORIRES,

- Data representation interface in the integrated GIS system,

- Analytical Internet Service for presenting results to the Internet [5-6].

Functional stages of DPOS operation:

1. Collection of various untyped data from open sources and Internet resources;

2. Loading information into an object-oriented database. Object structuring of collected data in OODB;

3. Presentation of information in the interface for generating and adjusting the parameters of mathematical models for the GAMS optimizer;

4. Launch of the integrated in DPOS optimizer GAMS, obtaining variants of mat-model solutions;

5. Use ready-made solutions in a table view or upload them back to the OODB for further processing;

6. Presentation of information in the interface of the GIS-system for building interactive maps, graphs and diagrams, with the possibility of retrospective and prospective data analysis;

7. Export of processed information to an external Analytical Internet Service in order to attracting interest of the scientific community to the results of research. 


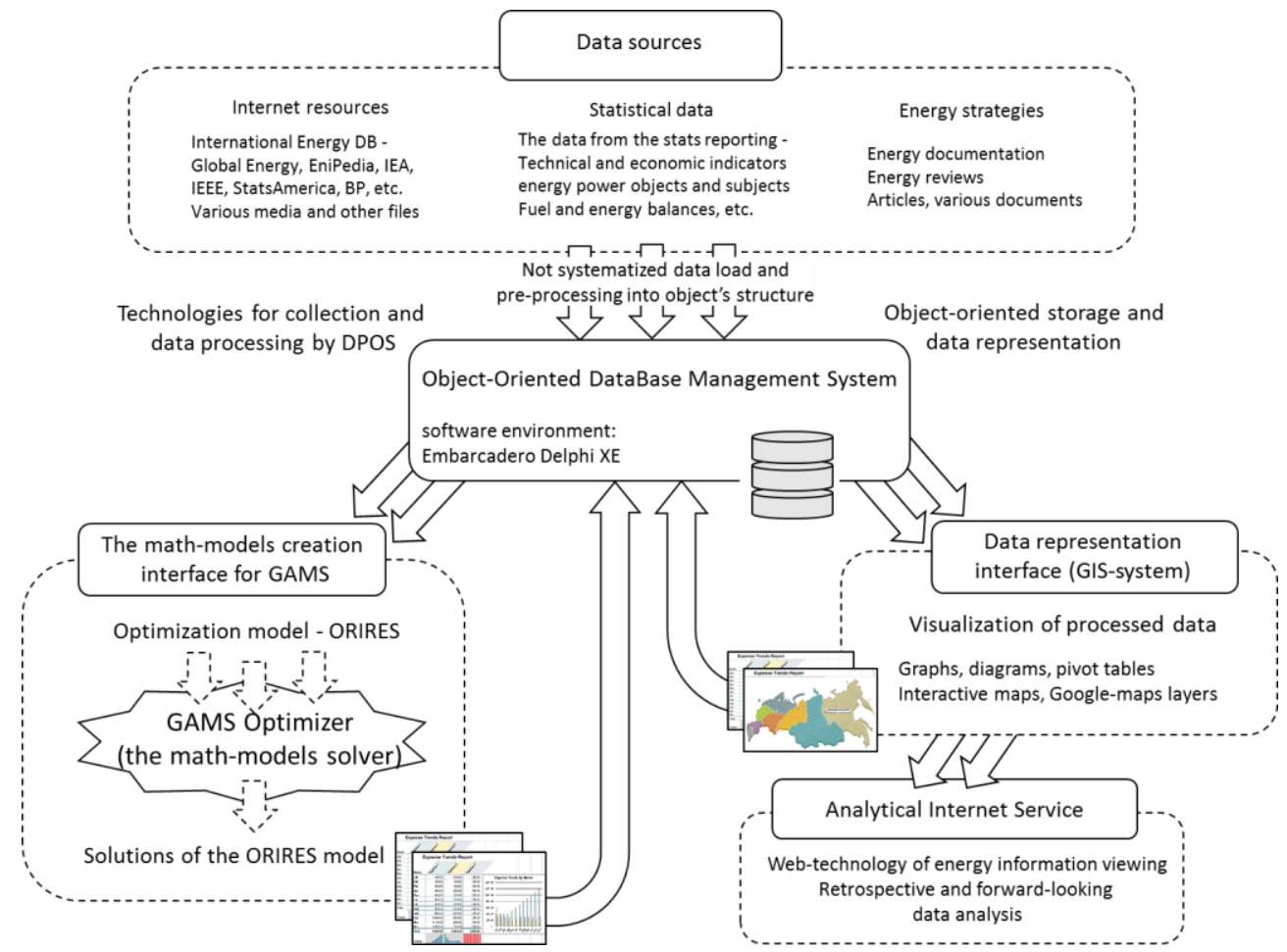

Fig. 1. Structural-functional scheme of the DPOS with integrated GIS-system and model ORIRES.

When implementing DPOS, modern mathematical, information and web technologies are used:

- The Embarcadero Delphi XE software environment, Google Maps API functions, SHP and XML files containing geographic and other information about objects of study are used as a basis for constructing the GIS-system and the Object-Oriented Database.

- To create a web application, modern web technologies based on PHP, Ajax, JQuery, JavaScript, MySQL, Bootstrap are applied.

- The forecast optimization model for the expansion of electric power systems ORIRES is written in the form of a set of mathematical formulas and equations solved through a specialized GAMS solver, the initial data for it, as well as the results of the solution can be obtained / loaded into an OODB for their subsequent processing and presentation.

\section{Storage and data processing in the Object-Oriented Database}

Official Internet sources of information about energy/power facilities and subjects of different countries have limited or paid access [7]. Own databases are not available for general use, and public information is mostly poorly structured for automatic machining.

To conduct qualitative research it is necessary to have the maximum completeness of information about the objects or processes being studied. In particular, in order to determine the initial state of the Interconnected Power System (IPS), it is necessary to have information about the installed and planned electricity generating capacities of this IPS. When collecting and obtaining such information, the researcher faces subjective and objective difficulties. Often sources of information are scattered and located on various Internet resources.

The authors of this work created a technology for collecting non-structured data and putting it in an object structure with subsequent verification and analysis. 
Feature of the technology for data collection and processing is the algorithm developed by the authors for obtaining data stored in the form of ontologies on specialized open sources. With the help of this algorithm, information about practically all energy/power objects in the world can be recorded in the Object-Oriented Database and then transferred to the object structure [8].

As a result, a thematic OODB is formed with a unique information gathered within a single structure. Its use greatly expands the possibilities and improves the quality of scientific research in this subject area.

For the DPOS programming interface, original procedures and algorithms for uploading data are developed, allowing users to work with information from OODB, analyze it, edit it and conduct various calculations. The calculation results can also be uploaded to the OODB for their subsequent use.

Objects in the OODB are represented as records with a set of parameters and values of the studied objects of different types, and have auxiliary media files that supplement information on objects: photos, articles or links to objects on the Internet. Objects in OODB can be various objects of the studied subject area: power plant, city, region, country, power interconnection, power line and others, Fig. 2.

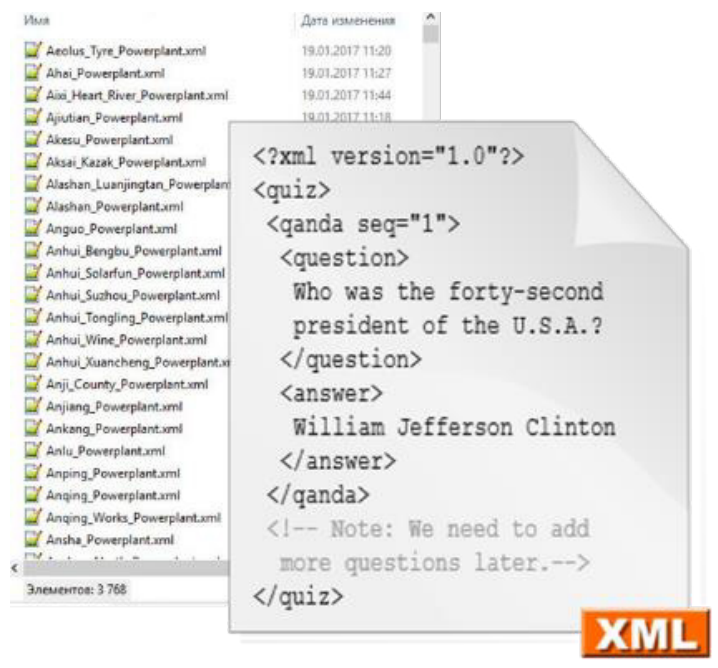

Fig. 2. An example of the content of object information in OODB in XML format.

Statistical information processing from OODB is carried out using a special query language that allows retrieving the parameters of objects and performing their sorting, sampling of

Objects have an extensible set of parameters that can be added while working with OODB. In particular, for the power plants, the parameters of their cartographic binding and the possibility of representation on maps in the GIS-system are added, Fig. 3. The shaded areas in the figure are the territories served by IPS of North China and IPS of Northeast China. 

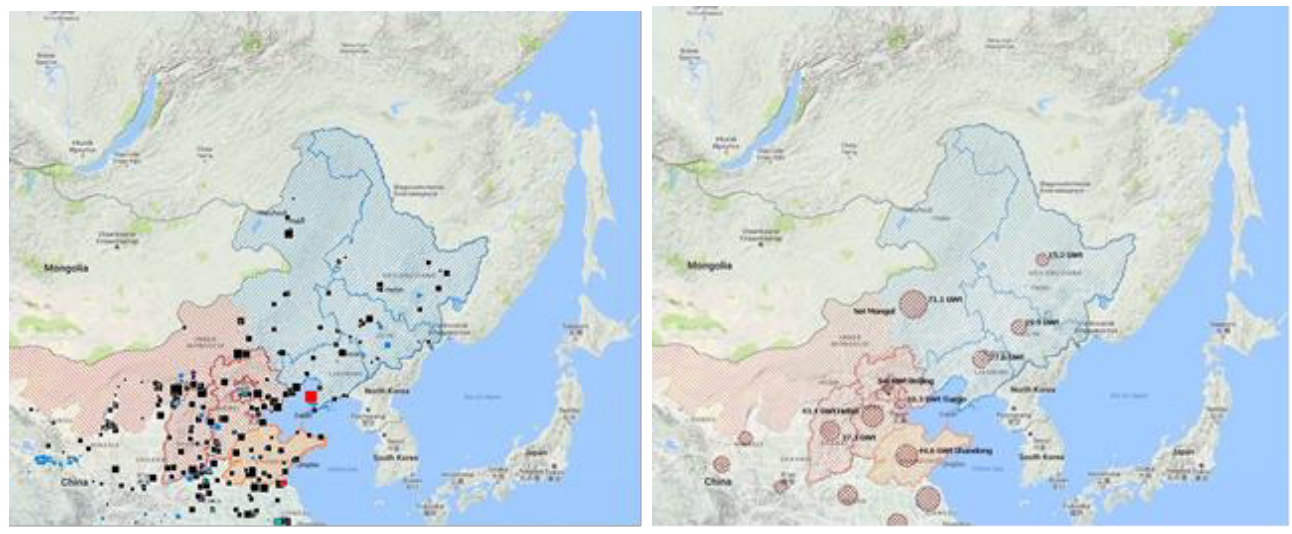

Fig. 3. Installed capacity of the North China IPS and North-East China IPS.

The collected information can be aggregated across different territorial and technological hierarchical levels [9] (country, IPS, etc.), which opens up opportunities for scaling the model - the creation of different levels of detail in the calculation scheme with different number of nodes and electrical connections. In Fig. 4 there is an example of a diagram for the Interstate Power Interconnection in the Northeast Asia region.

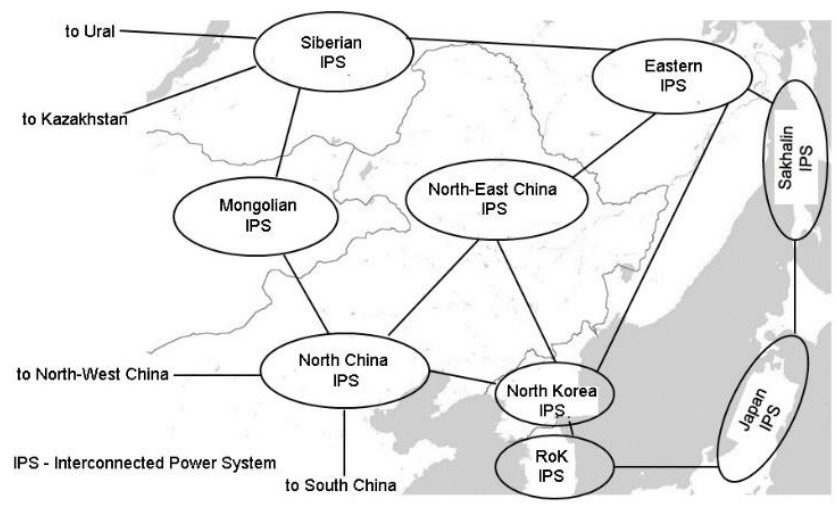

Fig. 4. The diagram of the Interstate Power Interconnection of Northeast Asia.

The primary list of objects in the OODB is formed from open sources on the Internet from EnergyPedia.org etc. [10]. IPS as well as individual power plants are represented in the OODB in the form of objects and their parameters. A number of other parameters have been introduced for this type of objects - daily load levels and graphs, aggregated volumes of power generating capacities by type of plants and so on. All OODB objects have numerical parameters and reference information, i.e. text parameters: name, province and others. The values of these parameters are used as input data integrated into the DPOS optimization model of ORIRES.

\section{The optimization model's solutions visualization in the DPOS interface}

The tasks of the ORIRES model include the calculation of various options for the formation of the IPS, the calculation of the optimal expansion of IPS, take into account capital investments, fuel costs, annual operating costs, variables that characterize intersystem flows, 
etc. [11]. The model solutions on the output from the mathematical optimizer GAMS are presented in a large amount of information on many pages, Fig. 5.

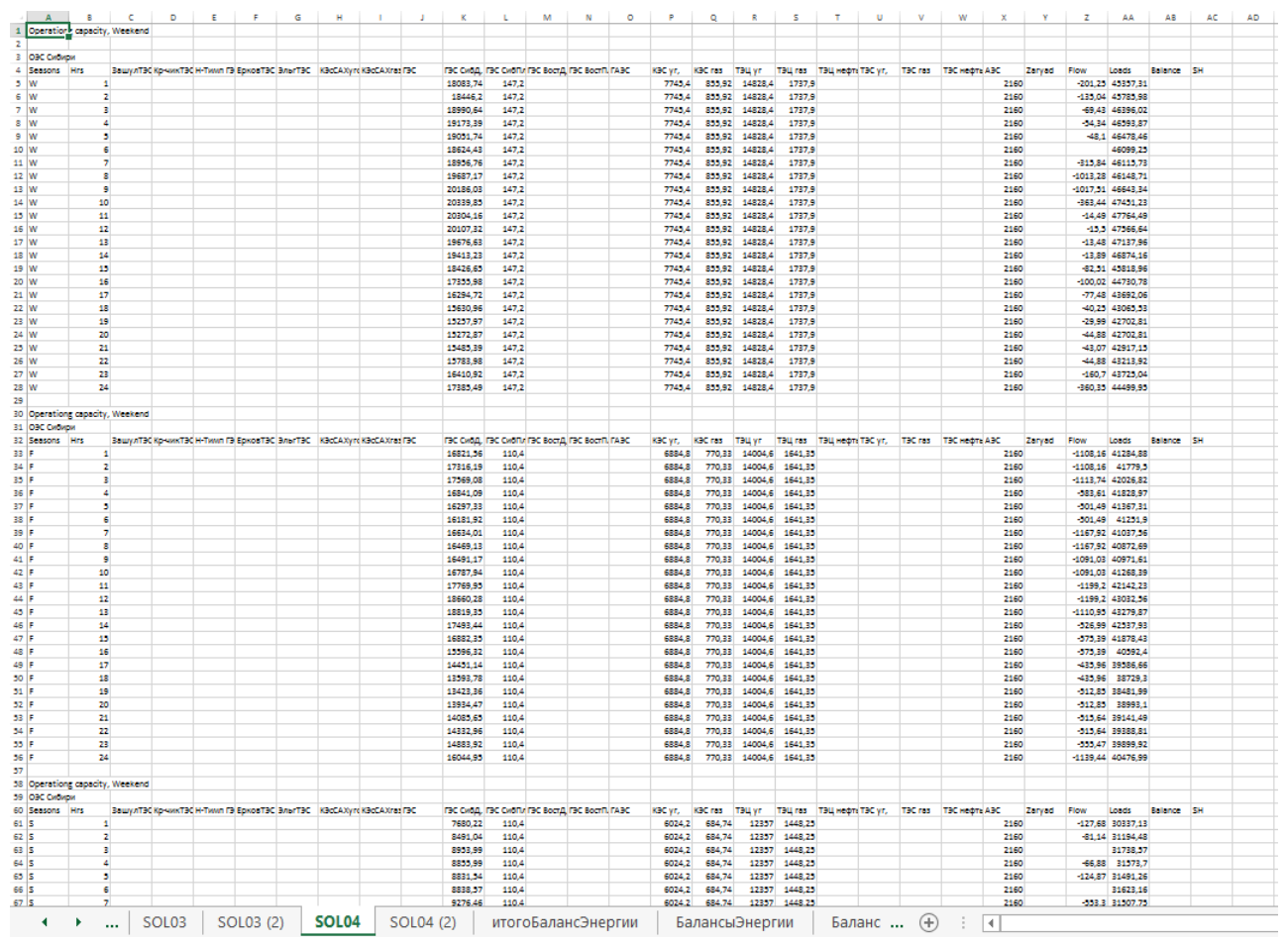

Fig. 5. Output data example of GAMS optimizer solution.

Multidimensional results of calculations are difficult to observe without a specialized graphical interface. To perceive this amount of information by an expert and his further analysis, specialized procedures are required to aggregate the results of the solution into different types of summary tables and diagrams.

The authors developed a special approach to step-by-step presentation of the results of the model solution. In the first step, the volumetric matrices of the primary detailed output data are converted into more aggregated tables of calculation results, which, while still sufficiently detailed, are convenient for analysis by experts. This form of visualization of the result of the optimization calculation is presented in the form of the annual energy and economic balance of South Korea's IPS, Table 1. In the balance sheet, exports are presented as a negative number, imports as positive. This table is only a small fragment of the presentation of the results of calculations in step 1.

Table 1. South Korea, Annual energy and economic balance, 2035.

\begin{tabular}{|c|c|c|c|c|c|c|c|}
\hline $\begin{array}{c}\text { Capacity } \\
\text { type }\end{array}$ & $\begin{array}{c}\text { Output, } \\
\text { GW- } \\
\text { hour }\end{array}$ & $\begin{array}{c}\text { Input, } \\
\text { GW- } \\
\text { hour }\end{array}$ & $\begin{array}{c}\text { Total, } \\
\text { GW- } \\
\text { hour }\end{array}$ & $\begin{array}{c}\text { Optimu } \\
\text { m } \\
\text { power, } \\
\text { MW }\end{array}$ & $\begin{array}{c}\text { Fuel } \\
\text { cost, } \\
\text { mln.\$ }\end{array}$ & $\begin{array}{c}\text { Service } \\
\text { cost, } \\
\text { mIn.\$ }\end{array}$ & $\begin{array}{c}\text { Total } \\
\text { cost, } \\
\text { mIn.\$ }\end{array}$ \\
\hline Nuclear & & 325968 & 325968 & 44000 & 2607 & 6504 & 9112 \\
\hline $\begin{array}{c}\text { Cogeneration } \\
\text { coal }\end{array}$ & & 7119 & 7119 & 1100 & 256 & & 256 \\
\hline Thermal coal & & 340408 & 340408 & 57100 & 12935 & 2384 & 15319 \\
\hline Thermal oil & & 3774 & 3774 & 1000 & 868 & & 868 \\
\hline
\end{tabular}




\begin{tabular}{|c|c|c|c|c|c|c|c|}
\hline $\begin{array}{c}\text { Cogeneration } \\
\text { gas }\end{array}$ & & 46402 & 46402 & 11000 & 6403 & & 6403 \\
\hline Thermal gas & & 107927 & 107927 & 33594 & 15109 & & 15109 \\
\hline Hydro & & 14117 & 14117 & 1900 & & 27 & 27 \\
\hline Renewable & & 2976 & 2976 & 4700 & & & \\
\hline $\begin{array}{c}\text { Total } \\
\text { generation }\end{array}$ & & 848695 & 848695 & 154394 & 38181 & 8916 & 47097 \\
\hline Storage & -3721 & & -3721 & & & & \\
\hline Total & $\mathbf{- 3 7 2 1}$ & $\mathbf{8 4 8 6 9 5}$ & $\mathbf{8 4 4 9 7 4}$ & $\mathbf{1 5 4 3 9 4}$ & $\mathbf{3 8 1 8 1}$ & $\mathbf{8 9 1 6}$ & $\mathbf{4 7 0 9 7}$ \\
\hline
\end{tabular}

In step 2, there is further aggregation and, most importantly, visualization of the output data in the form of graphs and diagrams, which is convenient for presenting results in reports and presentations. As an example, we consider a variant of plotting the graphs reflecting the hourly daily load in South Korea's IPS, meeting it by various types of generating capacities (nuclear, coal, gas, hydraulic, wind, etc.), as shown on the right-hand side of Fig. 6. The left part of Fig. 6 shows the columns representing the working and installed capacities, as well as limits on their expansion in the considered period (until 2035) for IPS of South Korea:

- Column 1 reflects the upper limit of how much capacity of power plants in a node can be increased compared to the current level.

- Column 2 reflects the "optimal" solution obtained as a result of the calculation by the ORIRES model, which meets the growing demand with minimal cost.

- Column 3 reflects the current mix of installed capacity.

- Columns 4 and 5 - the limits of the operating range in which the power output of each type of power plant can vary, which depend on their technical characteristics.

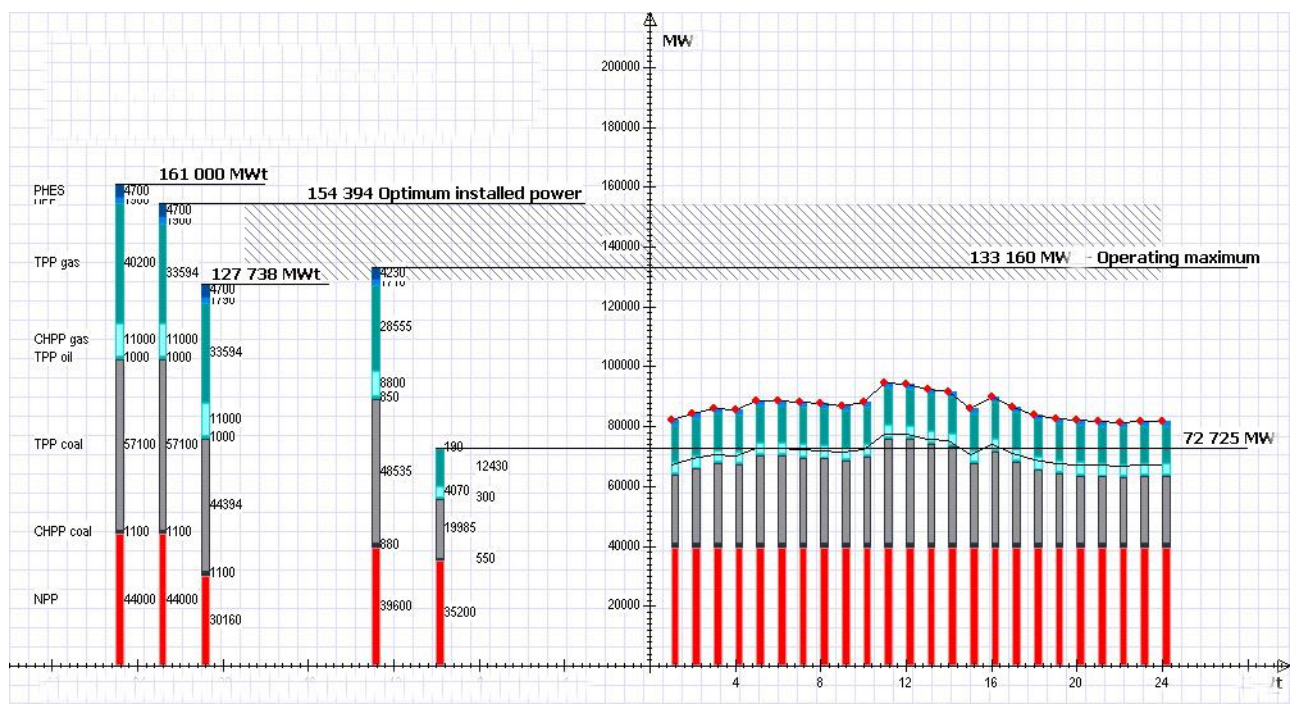

Fig. 6. Optimization of prospective installed capacities and hourly graph of meeting daily load by various types of power plants.

The diagram under the daily load graph shows how much power and what type of power plant was used for each hour. Usually considered are eight different types of daily load graphs (summer weekdays / holidays, autumn weekdays / holidays, winter weekdays / holidays, spring weekdays / holidays). Multiplying and totaling each of the eight daily generation of electricity by the corresponding number of days in a year, the amount of electricity produced 
per year is obtained. At the same time, the amount of electricity generated per year will be broken down into generation by types of power plants. In addition, the model calculates the cost of fuel, operational and investment cost of each type of power plant. Using it, we can get the final cost of generated electricity for the year.

The proposed approach to visualizing the results of the solution of the model increases the efficiency and convenience of perceiving a large amount of information aggregated and depicted in generalized graphs.

\section{Conclusion}

The authors developed the basic technologies for the construction of a specialized DPOS, including the procedures for collecting, storing and processing of poorly structured data, an interface for flexibly adjusting the parameters of the mathematical model ORIRES and its solutions by the GAMS optimizer, the presentation interface for retrospective and prospective analysis in the GIS system.

A new form of information presentation is proposed for more convenient comparison of different solutions of the optimization model using graphical visualization.

The DPOS proposed by the authors allows significantly increasing the efficiency of scientific research in the field of expansion of interstate power systems and improving the results of the solution qualitatively by using a convenient graphical interface with the ability to configure input and output parameters for the optimization model.

\section{References}

1. N.I. Voropai, S.V. Podkovalnikov, V.A. Savelyev, L.Yu. Chudinova, Electricity. Transmission and distribution, 3 (42), 8 (2017)

2. Douglas K Barry. Web Services, Service-Oriented Architectures, and Cloud Computing. Database Articles. Object-Oriented Databases (2013)

3. S.V. Podkovalnikov, V.A. Saveliev, L.Yu. Chudinova, Studies on Russian Economic Development, 26 (4), 403 (2015)

4. D. Gao, O. Khamisov, D. Sidorov, International Journal of Artificial Intelligence, 13, 120 (2015)

5. I.L. Trofimov, Energy Statistical Analytical Service. ESAS. World Energy on an interactive map (2015)

6. L.N. Trofimov, I.L. Trofimov, Energy Industry in Russia in 21 Century: Innovative development and management, 170 (Irkutsk, Russia, 2015)

7. Global Energy Observatory. Power Plants (2017)

8. I.L. Trofimov, Zbornik radova konferencije MIT 2013, 706 (Vrnjacka Banja, Serbia and Budva, Montenegro, 2014)

9. I.L. Trofimov, System research in the energy sector. Collection of works of young scientists ISEM SB RAS, 215 (Irkutsk, Russia, 2013)

10. Energy Pedia. Portal: Power Plants (2017)

11. L.S. Beliayev, S.V. Podkoval'nikov, V.A. Savel'yev, and L.Yu. Chudinova, Efficiency of Interstate Electric Ties (2008) 